\title{
Instrumental design of the Solar Observing Satellite: solar-C_EUVST
}

\section{Yoshinori Suematsu, Toshifumi Shimizu, Hirohisa Hara, Tomoko Kawate, Yukio Katsukawa, et al.}

Yoshinori Suematsu, Toshifumi Shimizu, Hirohisa Hara, Tomoko Kawate, Yukio Katsukawa, Kiyoshi Ichimoto, Shinsuke Imada, "Instrumental design of the Solar Observing Satellite: solar-C EUVST," Proc. SPIE 11852, International Conference on Space Optics - ICSO 2020, 118523K (11 June 2021); doi: 10.1117/12.2599610

SPIE Event: International Conference on Space Optics - ICSO 2021, 2021, Online Only 


\section{International Conference on Space Optics-ICSO 2020}

Virtual Conference

30 March-2 April 2021

Edited by Bruno Cugny, Zoran Sodnik, and Nikos Karafolas
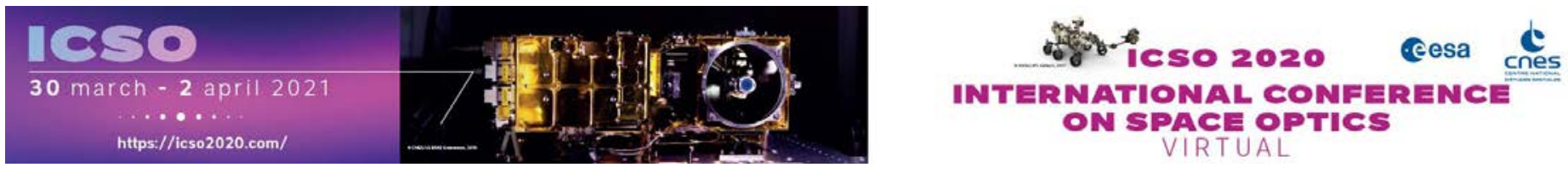

\section{Instrumental design of the Solar Observing Satellite: solar- C_EUVST}

\section{Cesa iso poresedings ecnes}




\title{
Instrumental design of the Solar Observing Satellite: Solar-C (EUVST)
}

Yoshinori Suematsu $^{* a}$, Toshifumi Shimizu ${ }^{b}$, Hirohisa Hara ${ }^{a}$, Tomoko Kawate ${ }^{c}$, Yukio Katsukawa ${ }^{a}$, Kiyoshi Ichimoto ${ }^{\mathrm{d}}$, Shinsuke Imada ${ }^{\mathrm{e}}$

${ }^{a}$ National Astronomical Observatory of Japan, 2-21-1 Osawa, Mitaka, Tokyo 181-8588, Japan ;

bISAS/JAXA, 3-1-1 Yoshinodai, Chuo-ku, Sagamihara, Kanagawa 252-5210, Japan;

${ }^{c}$ National Institute for Fusion Science, 322-6 Oroshi-cho, Toki, Gifu 509-5292, Japan ;

dAstronomical Observatory, Kyoto University, Kitashirakawa-Oiwake-Cho, Sakyo-Ku, Kyoto 6068502, Japan;

${ }^{\mathrm{e}}$ Institute for Space-Earth Environmental Research, Nagoya University, Furo-cho, Chikusa-ku, Nagoya 464-8601, Japan;

\begin{abstract}
The EUV High-Throughput Spectroscopic Telescope (EUVST) of Solar-C mission is a revolutionary spectrometer that is designed to provide high-quality and high cadence spectroscopic data covering a wide temperature range of the chromosphere to flaring corona to investigate the energetics and dynamics of the solar atmosphere. The EUVST consists of only two imaging optical components; a $28-\mathrm{cm}$ clear aperture off-axis parabolic primary mirror and a two-split ellipsoidal grating without a blocking filter for visible light before the primary mirror to achieve unprecedented high spatial and temporal resolution in EUV-UV imaging spectroscopic observations. For this reason, about $53 \mathrm{~W}$ of sunlight is absorbed by the multilayer coating on the mirror. We present an instrumental design of the telescope, particularly, primary mirror assembly which enables slit-scan observations for imaging spectroscopy, an image stabilizing tip-tilt control, and a focus adjustment on orbit, together with an optomechanical design of the primary mirror and its supporting system which gives optically tolerant wavefront error against a large temperature increase due to an absorption of visible and IR lights.
\end{abstract}

Keywords: UV imaging spectroscopy, solar space telescope, instrumental design of telescope

\section{INTRODUCTION}

The Solar-C (EUVST) is a mission of international collaboration led by JAXA ${ }^{[1]}$, which has been selected as the 4th satellite in the series of competitively chosen M-class mission to be launched with a JAXA Epsilon launcher in late 2026. The Solar-C will be launched into an altitude higher than $600 \mathrm{~km}$ of sun-synchronous polar orbit for continuous solar observations. The mission payload of Solar-C: EUV High-Throughput Spectroscopic Telescope (EUVST) is a unique spectrometer that aims to provide a conclusive answer to the most fundamental question in solar physics. The EUVST is designed to provide high-quality high cadence spectroscopic data covering a wide temperature range of the chromosphere $\left(210^{4} \mathrm{~K}\right)$ to flaring corona $\left(210^{7} \mathrm{~K}\right)$. The instrument should be able to achieve a throughput about ten times higher than ever owing to two-component optical configuration; a $28-\mathrm{cm}$ clear aperture off-axis parabolic primary mirror and a two-split ellipsoidal grating; each for specific UV and EUV range, both which have high reflective coatings in EUV-UV range $(17-21 \mathrm{~nm}, 47-125 \mathrm{~nm})$. In addition, a high spatial $(0.4 \mathrm{arcsec})$ and spectral resolutions require long focal length mirrors and gratings. Therefore, we need a spectroscopic telescope design which accommodate a long telescope/spectrograph configuration, satisfying a launcher condition and launch environment of JAXA Epsilon launcher. From the demands of high spatial resolution and imaging spectroscopy, the primary mirror needs to be light-weight and capable of active tip-tilt and image scanning control. From the request of high throughput, non-filtered sunlight strikes the primary mirror and so the temperature of the mirror could go up to $100{ }^{\circ} \mathrm{C}$ or so due to absorption of about $53 \mathrm{~W}$ of solar visible and IR lights. To attain high optical performance, an optomechanical design of the light-weight primary mirror and its supporting system is crucial, which can avoid mirror figure deformation due to mechanical and thermal stress. We present an instrumental design of the telescope, particularly, primary mirror assembly which enables slit-scan observations for imaging spectroscopy, an image stabilizing tip-tilt control, and a focus adjustment on orbit, together

*a yoshinori.suematsu@nao.ac.jp; phone 8142234 3713; fax 81422343700 
with an optomechanical design of the primary mirror and its supporting system which gives optically tolerant wavefront error against a large temperature increase due to an absorption of visible and IR lights.

\section{INSTRUMENTAL DESIGN OF EUVST TELESCOPE}

\subsection{Optical Design of EUVST}

The optical configuration of the EUVST spectrograph follows the two-element, magnified Rowland circle design pioneered by the Hinode/EIS ${ }^{[2]}$. This two-element design minimizes the number of reflections in the optical system to provide high throughput required to meet the scientific requirements. Figure 1 shows the optical layout and raytracing of EUV/UV light from the entrance aperture to the primary mirror, slit assembly, two-split grating, and focal plane detector arrays. The primary mirror is an off-axis parabola $(28 \mathrm{~cm}$ clear aperture, focal length $280 \mathrm{~cm}$ ), which makes a solar image on the slit. The primary mirror has a tip-tilt and image scanning mechanism and to minimize solar heat input on the primary mirror, a pupil of $28 \mathrm{~cm}$ aperture is placed just in front of the primary mirror (Figure 2). The slit selects a one-dimensional portion of the solar image from the primary mirror. The slit assembly has a function to select four kinds of slit; $0.1 \times 300,0.2 \times 300,0.4 \times 300$, and $1.6 \times 300$ arcsec. This light is incident onto an ellipsoidal diffraction grating that is segmented into two halves, one each for the short $(17-21 \mathrm{~nm})$ and long $(47-125 \mathrm{~nm})$ wavelength portions of the spectral range. The highly corrected off-axis ellipsoidal gratings with variable line spacing provide high image quality at the focal plane across all wavelength ranges ${ }^{[3]}$.

The gratings are figured to optimize the image quality and efficiency of each passband. The highly magnifying optics allows an instrument of reduced size compared to that of a conventional spectrometer with similar spatial resolution. This grating unit will have focus adjustment along the telescope chief ray to make the best-focused spectra on the detector surfaces; CCD for EUV band and three intensified active pixel sensors (IAPS) for UV band.

The light reflected from the slit plane is transferred by two folding mirrors with dielectric coatings that reflect the desired UV lights around $280 \mathrm{~nm}$ and transmit the rest of the visible and IR light to heat dumps behind them (slit-jaw imager). A collimator and camera lens of fused silica relay the image to the detector through a narrow passband Solc-type birefringent filter like aboard IRIS ${ }^{[4]}$. The imager provides context chromospheric (Mg II h\&k lines) and photospheric images.

To protect the slit and the slit-jaw imager from excessive solar heat load, a pre-slit is placed just in front of the slit. The pre-slit transmits a clear field-of view (FOV) of 320x320 arcsec and absorbs the solar light outside the FOV up to 1.4 solar radii off-set pointing. The pre-slit consists of a blackened disc of $86 \mathrm{~mm}$ square located $200 \mathrm{~mm}$ ahead of the slit and has a squared cone starting at its center tapering from $18.6 \mathrm{~mm}$ to $9.4 \mathrm{~mm}$ at $20 \mathrm{~mm}$ ahead of the slit. It is expected to intercept $35 \mathrm{~W}$ of heat from the primary mirror. The pre-slit is conductively connected to a radiator near the entrance to dump the heat absorbed into space. Nearly identical design concept except for a coating on top will be used to the Heat Dump Unit of Hinode/SOT which has been perfectly working in space over more than 13 years. A detailed design of the pre-slit is given in Figure 3.

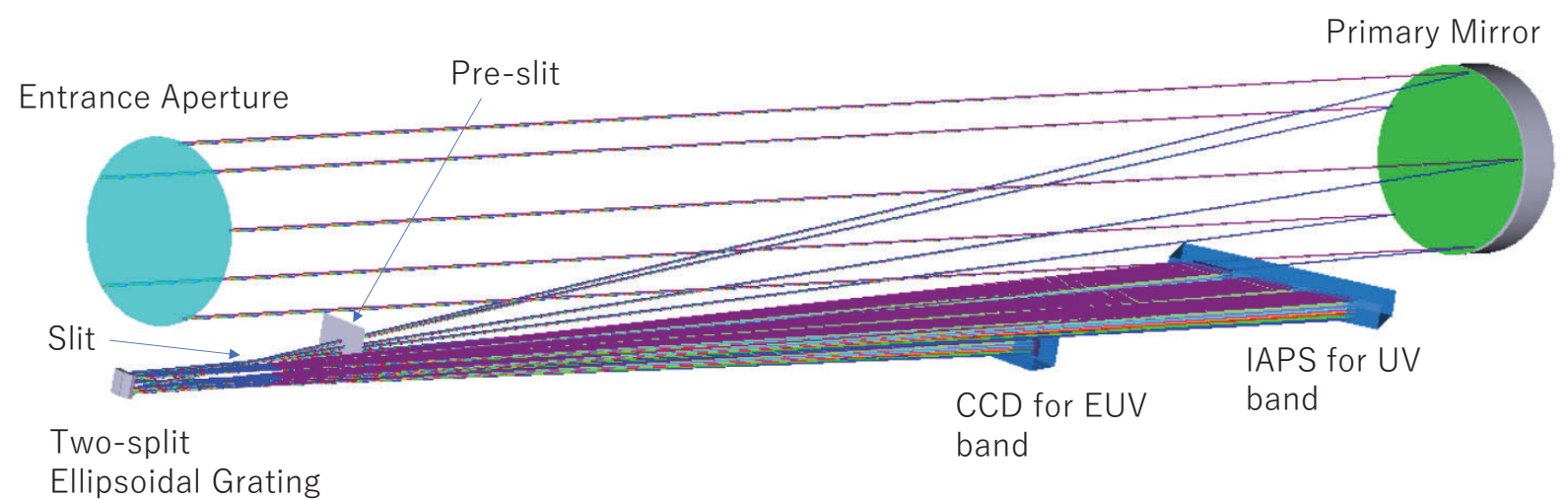

Figure 1. Schematic telescope and spectrograph optical layout of Solar-C_EUVST. Slit-Jaw Imager is not shown. 


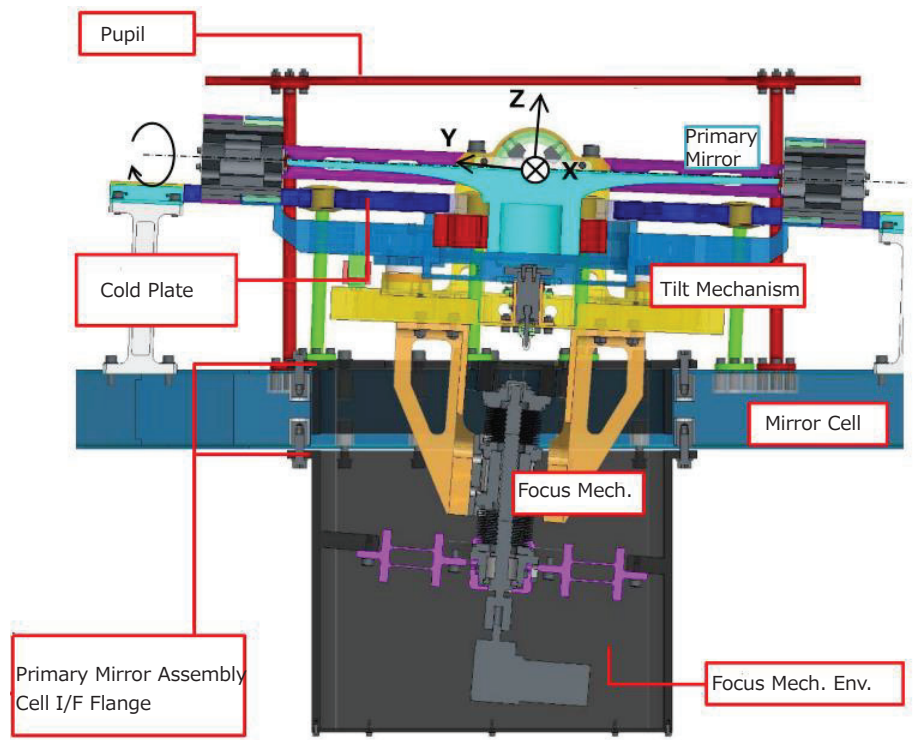

Figure 2. Conceptual design of primary mirror assembly. It consists of a two-axis gimbal mechanism for slit-scan and image stabilization tip-tilt mechanism; tilt stabilization: $0.06 \operatorname{arcsec}(3 \sigma)$ with a band width of $10 \mathrm{~Hz}$, the tilt resolution: 0.02 arcsec and the stroke larger than $\pm 80 \mathrm{arcsec}$, and a translational actuator for focus adjustment; the resolution of $0.01 \mathrm{~mm}$ and the stroke of $\pm 5 \mathrm{~mm}$

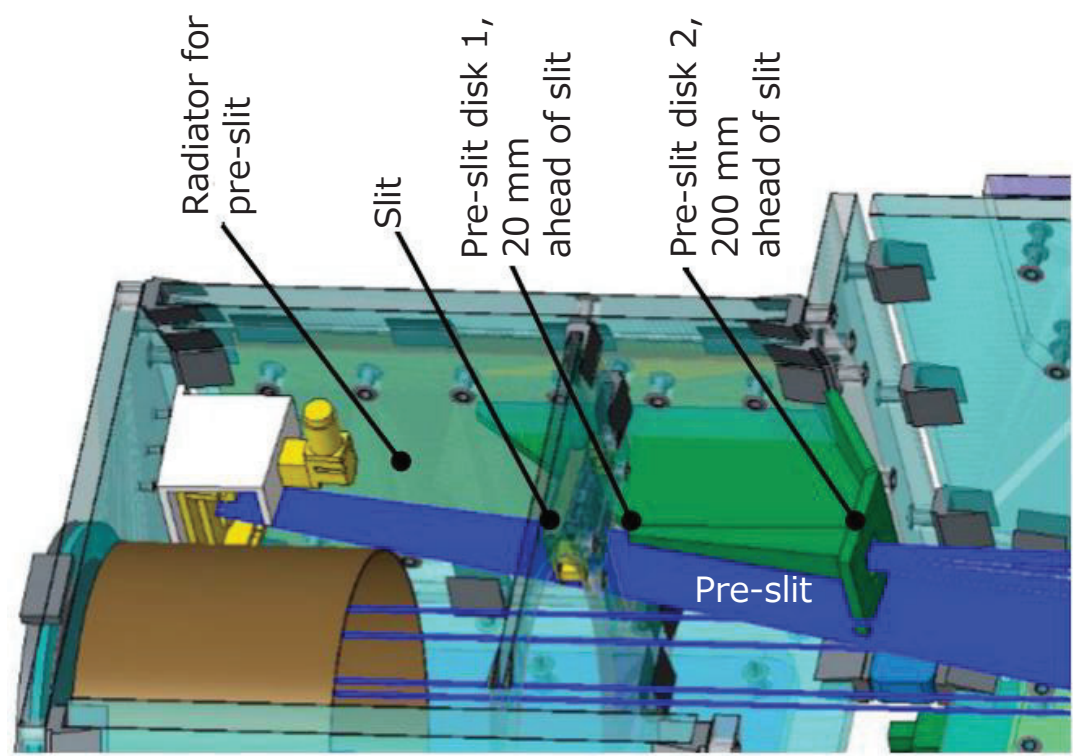

Figure 3. The pre-slit consists of a blackened disc of $86 \mathrm{~mm}$ square located $200 \mathrm{~mm}$ ahead of the slit and has a squared cone starting at its center tapering from $18.6 \mathrm{~mm}$ to $9.4 \mathrm{~mm}$ at $20 \mathrm{~mm}$ ahead of the slit.

\subsection{Structure Design of EUVST}

The EUVST instrument consists of two sections; a telescope section and a spectrograph section (Figure 4). The entire package is to be mounted to a mechanical interface at the top panel of the satellite bus module. The telescope section consists of an aperture door, the pre-slit heat dump unit, the slit-jaw imager, the primary mirror assembly with image stabilization and image scanning capabilities. The spectrograph section houses a slit assembly, a grating assembly, focal 
plane assemblies, CCD electronics box, the intensified active pixel sensors (IAPS) electronics box and the radiator for the detectors.

It is noted that the EUVST instrument is basically designed as a passive, cold biased thermal system, optimized for insensitivity to external heat sources. The thermal design draws strong heritage from the spectrometers on SoHO/SUMER ${ }^{[5]}$ and Hinode/EIS ${ }^{[2]}$, where experience has shown that an ultra-stable temperature equilibrium of the optical bench is required to reach high optical performance characteristics. Long-term variations (seasonal, aging effects) are less critical. These requirements put severe constraints on the thermo-mechanical bus interface and call for an isostatic mount of the instrument to prevent thermo-elastic cross-effects and to minimize radiative and conductive coupling to the bus. In addition, the sun-synchronous orbit should provide a relatively stable thermal environment. The varying effects of Earthshine and Albedo on the instrument are minimized by optimal radiator placement and orientation.

Detector Assemblies: A CCD for shorter wavelength and IAPSs (intensified active pixel sensor) for longer wavelength require mechanical stability, thermo-elastic insusceptibility, cooling $\left(<-60^{\circ} \mathrm{C}\right.$ for the $\mathrm{CCD}$ and $<-45^{\circ} \mathrm{C}$ for the IAPSs) and optimized accommodation of the proximity electronics. These requirements will be achieved by an advanced design that is based on Hinode/EIS experience. The CCD will be thermally isolated from the structure and cooled using a flexible strap to an external radiator. The IAPS will be cooled using an additional external radiator.

An estimated total weight of the EUVST instruments is about $235 \mathrm{~kg}$ and the first eigen frequency in $\mathrm{x}$ - and $\mathrm{y}$-direction is $36.8 \mathrm{~Hz}$ and in z-direction is larger than $100 \mathrm{~Hz}$, which fulfills the requirements from the launch condition.

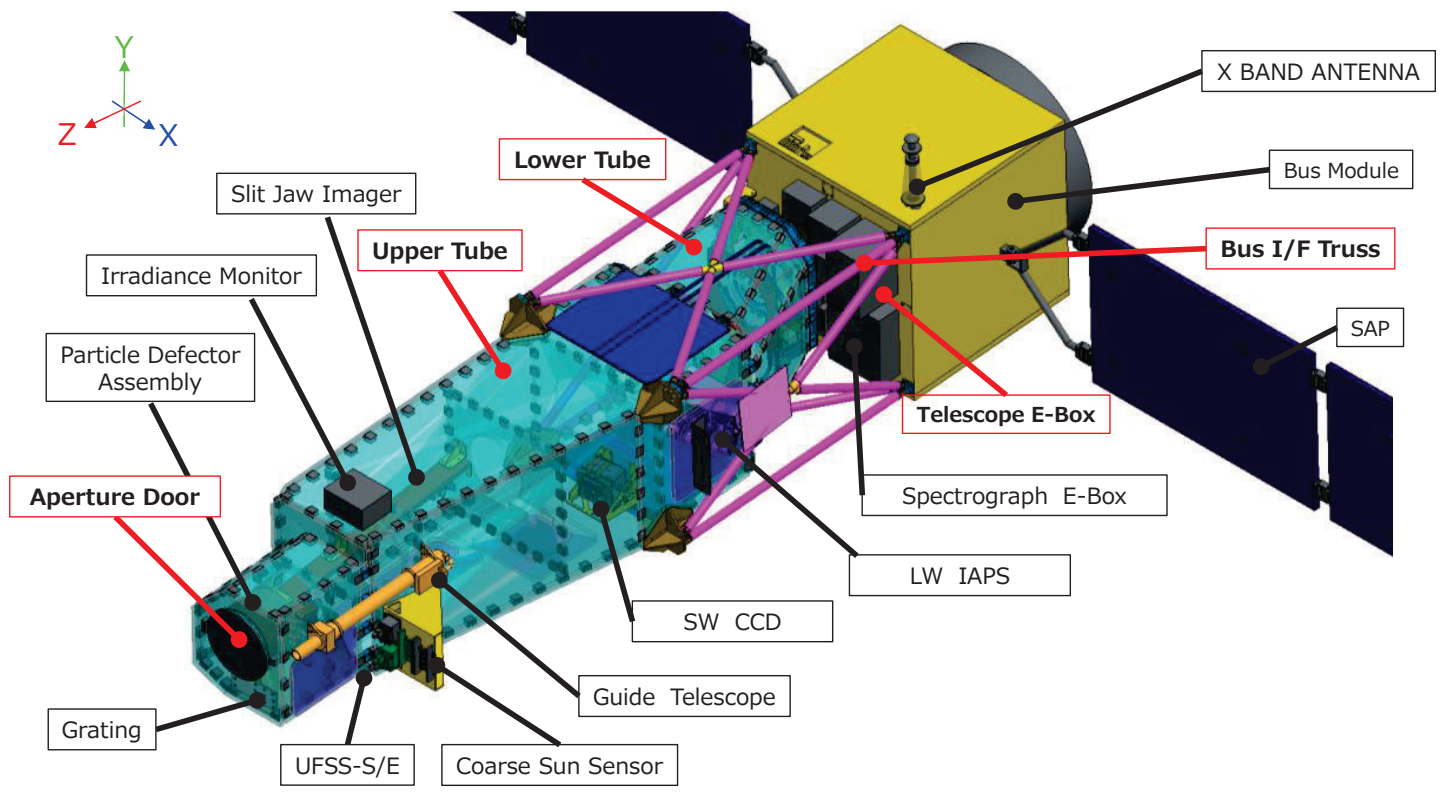

Figure 4. Schematic structural design of the Solar-C (EUVST) whose components are configured into an integrated telescope-spectrograph structure.

\subsection{Thermal Design of Primary Mirror Assembly}

One of the key areas in the EUVST instrument is the primary mirror assembly; the primary mirror mounted on a tip-tilt and scanning mechanism and focusing mechanism. A schematic design of the primary mirror assembly is shown in Figure 4. It has a light-weighted (target of $2 \mathrm{~kg}$ ) $30 \mathrm{~cm}$ diameter off-axis paraboloid mirror with a highly reflective coating at the observing EUV/UV wavelength. It contains key technologies for achieving the required spatial resolution,

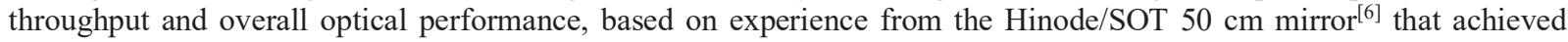
much higher spatial resolution than EUVST. The primary mirror is fabricated from Zerodur or ULE for their ability to achieve very low surface micro-roughness and its ultra-low thermal deformation. A micro-roughness of $<0.5 \mathrm{~nm}$ RMS will be achieved based on the experience in fabricating the $20 \mathrm{~cm}$ Zerodur mirrors for SDO/AIA ${ }^{[7]}$. 
The tilt stage underneath the mirror consists of a two-axis gimbal mirror cell for the tip-tilt image stabilization and the image scanning observations, allowing to scan the field of view by moving step by step the position of the solar image on the slit. The tilt stage will be driven with four voice-coil actuators which have been used in previous projects mounted on the mirror cell. Since the mirror cell is loose when power is off, a launch-lock system for the mirror cell is equipped.

The whole mirror cell unit including the primary mirror is supported by a translational actuator like a ball-screw which is used for re-focusing on orbit. In this case, the mirror cell needs to be additionally supported by leaf springs or guide rails. A prototype of the focus mechanism unit had been developed and qualified for usage in the space environment for the EUVST. It used a ball screw to translate the motor motion to the linear motion and was designed to have a step resolution of $100 \mu \mathrm{m}$ in the range of $60 \mathrm{~mm}$. In the case of EUVST, considering a dehydration contraction of the CFRP structure and a focal depth of the primary mirror, the focus mechanism will have a stroke of $\pm 5 \mathrm{~mm}$ with a resolution of $10 \mu \mathrm{m}$.

The light-weight structure of primary mirror and its mounting is one of the most critical parts in the primary mirror assembly. Our heritage is the mounting mechanisms for the $50 \mathrm{~cm}$ primary and $16 \mathrm{~cm}$ secondary mirrors used in Hinode/SOT ${ }^{[6]}$. From the consideration of light-weight and thermal deformation issues, the primary mirror and its supporting mechanism for EUVST was designed based on the secondary mirror of SOT, which has a shape like a large Japanese flat wine cup and is supported by a super-invar tripod-shaped pad of stress relief spring legs at the side of the mirror's backside cylindrical hump.

By advancing the design of SOT secondary mirror and taking advantage of newly developed ultra-low CTE metal: a zero-invar, the primary mirror and its supporting system for EUVST was designed, which is optically tolerant for a large temperature increase. The primary mirror shaped like the flat wine cup, made of ultra-low CTE glass material, is supported by three stress-free mounting mechanisms (zero-invar-made bipods) bonded on the side of the mirror's backside cylindrical hump. The bipods are rooted in a cylindrical hole of a tilt stage of rotational mechanism. The three bipod mechanisms provide a kinematic mount for the primary mirror and avoid stresses to the mirror resulting from dimensional errors in machining and temperature changes.

We have been studying a thermal design of telescope section, particularly the primary mirror assembly, using the expected solar heat absorption by optical components. From the request of high throughput, non-filtered sunlight strikes the primary mirror and so the temperature of the mirror surface could be very high due to absorption of about $53 \mathrm{~W}$ of solar visible and IR lights. On the other hand, the temperature of the primary mirror bonding part and underlying tip-tilt and slit-scanning mechanisms should be well lower. To cool the primary mirror, a cold plate is placed underneath the mirror which is connected with a radiator by heat pipes. Modeling of the current primary mirror assembly design (Figure 5) indicates that the temperature goes up to as high as $110^{\circ} \mathrm{C}$. To have a smaller thermal deformation of the primary mirror, thermal design is required to keep the temperature gradient between the mirror surface of the primary mirror and the bottom of the ribs below $30{ }^{\circ} \mathrm{C}$, using a heater control at mirror supporting system. As a result, the wavefront error below $10 \mathrm{~nm}$ RMS can be realized and the margin large enough to the 0.4 arcsec spatial resolution budget. 


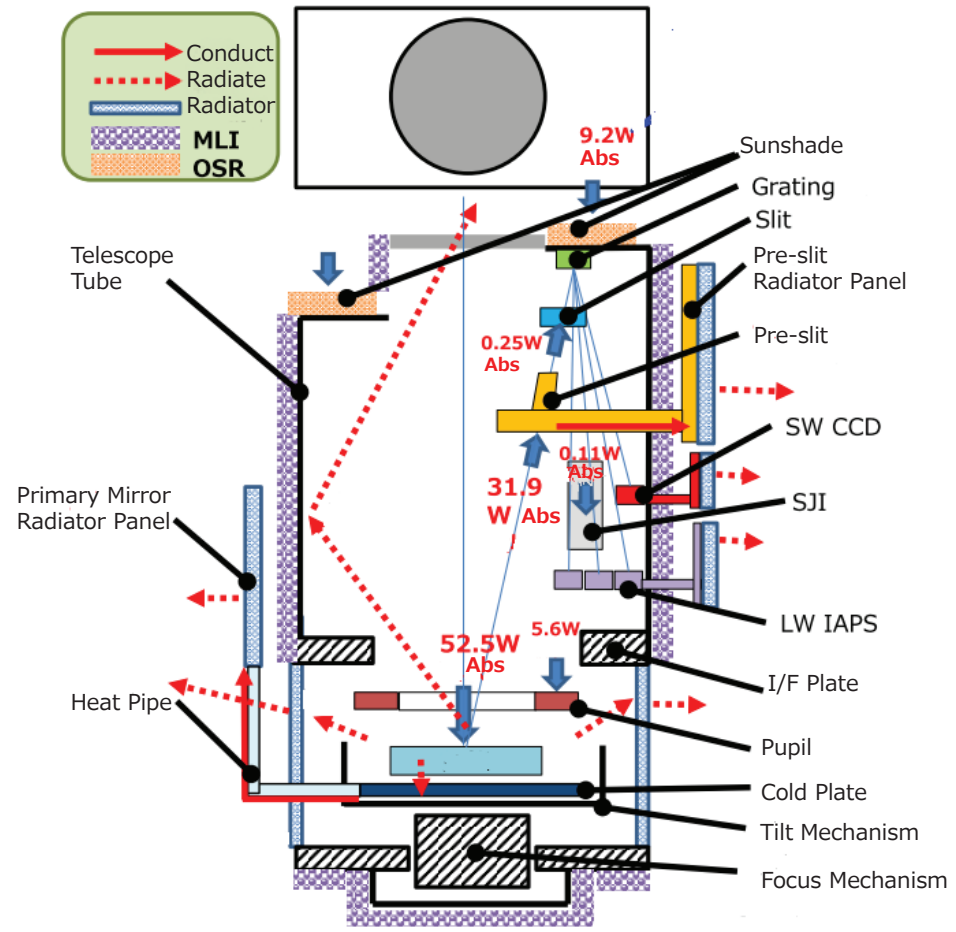

Figure 5. Thermal design of the primary mirror assembly and expected temperature distribution of structure around the primary mirror.
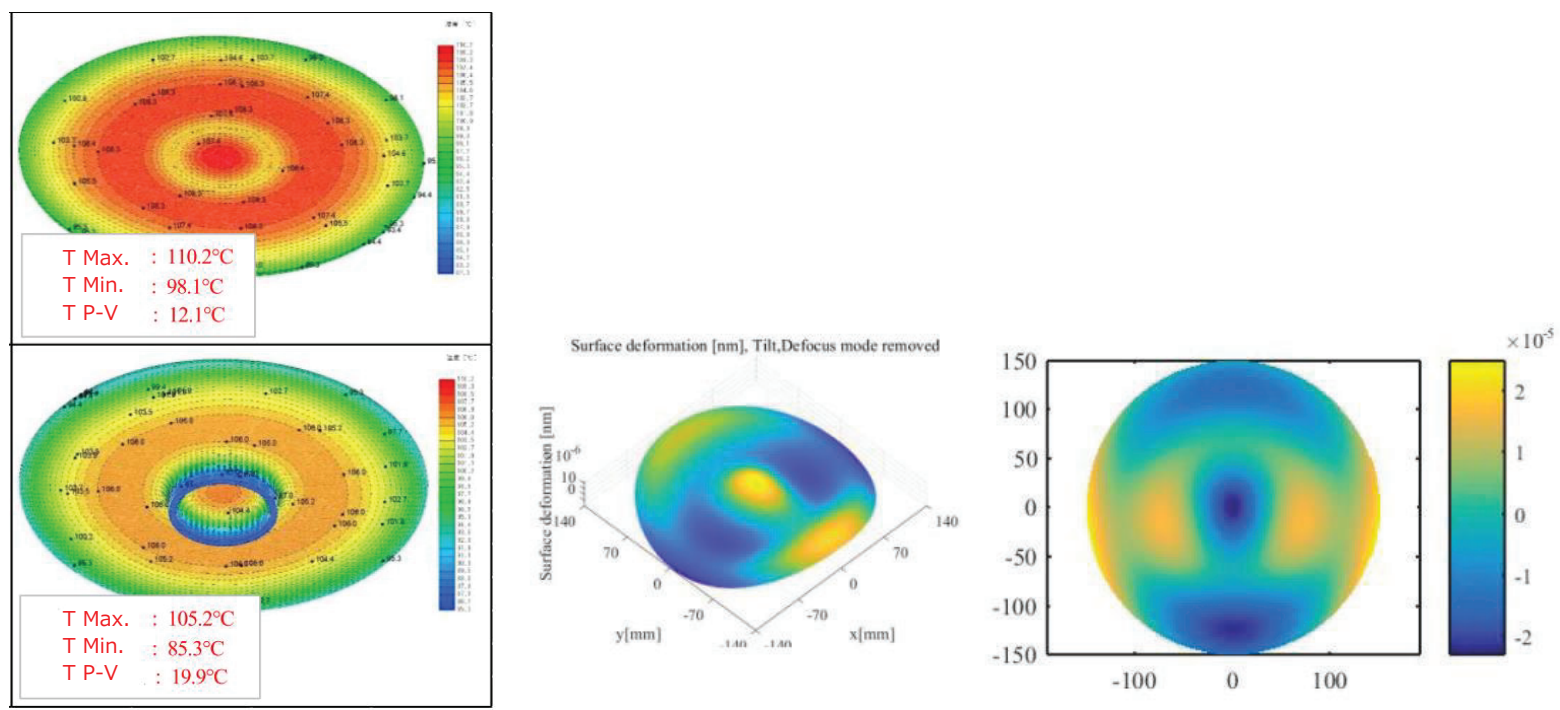

Figure 6. Temperature map (left) of primary mirror in the case of heater control at mirror support pads from the thermal model in Figure 5; the surface temperature is $\min 98^{\circ} \mathrm{C}$ and $\max 110^{\circ} \mathrm{C}$, and corresponding wavefront error map of the primary mirror (right) including the effect of stress from the mounting system; the wavefront error is $9.6 \mathrm{~nm}$ RMS. 


\section{SUMMARY}

The Solar-C (EUVST) is designed to provide high-quality high cadence spectroscopic data covering a wide temperature range of $210^{4} \mathrm{~K}$ to $210^{7} \mathrm{~K}$. The EUVST instrument enables to achieve a throughput about ten times higher than ever before using two-component optical configuration; a 28-cm clear aperture off-axis parabolic primary mirror and a twosplit toroidal grating. The high spatial $(0.4 \mathrm{arcsec})$ and spectral resolutions require long focal length mirrors and gratings. We presented a structure and thermal design of telescope, particularly the primary mirror assembly; to realize high spatial resolution and imaging spectroscopy, the primary mirror is mounted on an image stabilization tip-tilt and scanning mechanism and focusing mechanism. Since non-filtered sunlight strikes the primary mirror, the temperature of the mirror surface could be very high due to absorption of about $53 \mathrm{~W}$ of solar visible and IR lights. However, the temperature of the primary mirror bonding part and underlying tip-tilt and slit-scanning mechanisms should be well lower. Modeling of the current primary mirror assembly design indicates that the temperature goes up to as high as $110{ }^{\circ} \mathrm{C}$. To have a smaller thermal deformation of the mirror, thermal design is required to keep the temperature gradient between the mirror surface of the primary mirror and the bottom of the ribs below $30{ }^{\circ} \mathrm{C}$, using a heater control at mirror supporting system. As a result, the wavefront error below $10 \mathrm{~nm}$ RMS can be realized and the margin large enough to the 0.4 arcsec spatial resolution budget.

\section{REFERENCES}

[1] Shimizu, T., Imada, S., Kawate, T. et al., "The Solar-C (EUVST) mission: the latest status", Proc. SPIE 11444, id. $114440 \mathrm{~N}(2020)$.

[2] Culhane, J. L., Harra, L. K., James, A. M., et al., "The EUV Imaging Spectrometer for Hinode", Solar Phys., 243, 19-61 (2007).

[3] Kawate, T., Tsuzuki, T., Shimizu, T., et al., "A sensitivity analysis of the updated optical design for EUVST on the Solar-C mission", Proc. SPIE 11444, id. 114443J (2020).

[4] De Pontieu, B., Title, A. M., Lemen, J. R., et al., "The Interface Region Imaging Spectrograph (IRIS)", Solar Phys., 289, 2733-2779 (2014).

[5] Wilhelm, K., Curdt, W., Marsch, E. et al., "SUMER - Solar Ultraviolet Measurements of Emitted Radiation", Solar Phys., 162, 189-231 (1995).

[6] Suematsu, Y., Tsuneta, S., Ichimoto, K., et al., "The Solar Optical Telescope of Solar-B ( Hinode): The Optical Telescope Assembly", Solar Phys., 249, 197-220 (2008).

[7] Lemen, J. R., Title, A. M., Akin, D. J. et al., "The Atmospheric Imaging Assembly (AIA) on the Solar Dynamics Observatory (SDO)", Solar Phys., 275, 17-40 (2012) 